\title{
Moving Vehicle Detection: A Review
}

\author{
S.P.Patil \\ Assistant Professor \\ Dept.Comp. Science Engg. \\ G.H.R.I.E.M, Jalgoan
}

\author{
M.B.Patil \\ PG Scholars \\ Dept.Comp. Science Engg. \\ G.H.R.I.E.M, Jalgoan
}

\begin{abstract}
Moving vehicle detection is an essential process for Intelligent Transportation system. During the last decade, a large amount of work has been trying to produced output for this challenge; however, performances of most of them still fall far behind human perception. In this paper the object detection problem is studied, analyzing and reviewing the most important and newest techniques. We propose a classification of all these techniques into different categories according to their main principle and features. Moreover, study and point out their proposed methods, weather condition mentioned for the proposed methods and some other conditions like as jamming, shadow effects on the vehicles.
\end{abstract}

\section{Keywords}

Vehicle detection, jamming, traffic monitoring

\section{INTRODUCTION}

With the increasing vehicles on the road there is need to develop a system which provides information of vehicles to the driving assistant system in intelligent transportation system. Moving vehicle detection is the subpart of object detection. It is an essential building block for traffic monitoring and many other applications. Most of the research tries to tackle the problems related to vehicle detection and make the system able to find out or detects moving and stable vehicles in various weather conditions. However, despite the vast literature, performances of most of the algorithms still fall far behind human perception. In this literature, the study is depend upon different attributes like as weather conditions(Day,Night,Rainy,Cloudy),the used method in the reference paper is able to removed shadow effects or not, able to handle jamming or not and at last what is the disadvantage of used method by the researchers. Attributes used for review work are summarising as follows:-

- Weather condition mentioned:-There is various weather conditions as we all are known. In this literature survey four weather conditions are mentioned (Day, Night, Rainy, and Cloudy).For e.g., if Day time condition is mentioned by the researchers then in Table 1 contains 'Yes' otherwise 'No' in weather condition columns.

- Vehicles Detected in Jamming:-Jamming is also the condition in which traffic is occurred on the road. If this attribute is handling in previous research then write 'Yes' in the following Table 1.

- Shadow Effect Remove:-This also the condition where the vehicle is detected under shadow or the previous method removes shadow effect or not. If previous method detect vehicle under this condition then Table 1 contains 'Yes' otherwise 'No'.

- Disadvantage:-This attribute shows the disadvantage in previous research paper.
This paper is organized as follows. Section II addresses the literature survey. Conclusion is presented in Section III.

\section{LITERATURE SURVEY}

With the growing number of vehicles, traffic information increasingly becomes important for drivers. Many approaches have been proposed for tackling related problems in intelligent transportation system (ITS). Wang [1] proposed this model for two reasons. First is to formulate contextual dependencies in road traffic scenes based on conditional random field. The second is to develop a real-time algorithm of moving vehicle detection with cast shadow removal for automatic traffic monitoring. It could handle moving cast shadows lights and various weather conditions. But the problem related to JRF model is, it is not able to classify vehicles and dies not find out velocity also.L.W. Tsai, J.-W. Hsieh and K.-C Fan presented a approach for detecting moving vehicles using two parameters (colour and edges) from static images. This proposed method creates a new transform model for finding out vehicle candidate related to their colours in various environments but in traffic jams or overlapping of multiple vehicles conditions it does not able to detect vehicle candidates[2].

Zhang et al. [3] developed a multilevel framework to detect and handle vehicle occlusion. The proposed framework composed of three levels which are as intraframe, interframe, and tracking levels to resolve the occluded vehicles. In first level i:e intraframe level it evaluates the compactness ratio and interior distance ratio of vehicles, in interframe level, Implements the subtractive clustering on the motion vectors of vehicles, and the occluded vehicles are separated according to the binary classification of motion vectors. In tracking level occluded vehicles are tracked by using a bidirectional occlusion reasoning mechanism. In [4] Neeraj analyze the congested highway situations because single vehicles either occlude each other or are connected together by shadow. Moreover, scenes contain noise in the traffic monitoring videos because of sunny, rainy, cloudy, nighty weather conditions. This method can separate individual vehicles from occluded vehicles by tracking feature points movements and assigns to the motion vector for over-segments fragmentations.

Melo [5] proposed a method which will play important role in highway monitoring and road management schemes. This method addresses the problem related to lane detection and that will classify vehicles through the analysis using standard installation of traffic surveillance cameras. It described a lowlevel object tracking system that produced accurate vehicle motion trajectories, which could further be analyzed to detect lane centers and classify lane types.

In that system, the moving vehicles are handled using lanedetection method. They marked the lane using color which is not affected by illumination changes and the lane where the space is for vehicles. If any vehicle is found same colors as the lane marks, that vehicle will be detected by the three 
parameters which is as size, shape and motion information. This mechanism finding out the right and left boundaries of the lane and effectively eliminates the influence of passing vehicles [6].

A new background subtraction algorithm based on the sigmadelta filter, which was intended to be used in urban traffic scenes. Colour information is not use in that model. The sigma-delta background estimation algorithm provides a recursive computation of a valid background model of the scene, assuming that, at the pixel level, the background intensities are present most of the time. However, this model quickly degrades under slow or congested traffic conditions, due to the integration in the background model of pixel intensities belonging to the foreground vehicles [7].

An example-based algorithm for moving vehicle detection will be composed of Background Estimation, Block Division and Candidates Selection, Feature Extraction, SVM-Based Classification, Shape Representation. In background estimation steps it separates the background from its foreground based on interframe difference or the subtraction of an input image from a reference image. The second step, Block Division and Candidates Selection input image is divided into same size nonoverlapped blocks. The third block feature extraction extract the features of vehicles and after that classify the vehicles by using SVM classifier.PCA method is used to extract and reduced the size of original images[8].

Cucchiara et al. [9] proposed method based on artificial techniques. This method detects the vehicles from the visual data by using rule based reasoning. Traffic data may come from different sensors such as loop detectors, pneumatic sensors, or cameras. In particular, in the area of urban traffic monitoring, real-time data are integrated with short- and longterm knowledge of the traffic status, to dynamically update traffic information. This approach are mixing of two levels one is low level processing model and another one is high level processing module. The frames of images are described as day frame and night frame the both frames are input to the low level processing. In low level, the processing on two frames that means night and day frame are different and for that two different conditional inputs the parameters measured by the model is different. In night frame the parameters or steps are morphological analysis and headlight pairing. In the day frame the steps are motion detection, luminance variation detection and moving edge detection. After processing of input by referring different parameters, the output of low level provided to high level processing module. The high-level module is designed as a forward chaining production rule system, working on symbolic data, i.e., vehicles and their attributes (area, pattern, direction, and others) and exploiting a set of heuristic rules tuned to urban traffic conditions. The artificial techniques on the base of rule based reasoning finding out the vehicles in urban traffic scenes.

Álvarez et. Al. proposes the method to detect the road by using onboard camera. So for that purpose they defined road detection algorithm. Road detection is of high relevance for autonomous driving, road departure warning, and supporting driver-assistance systems such as vehicle and pedestrian detection. This method detects the pixels which are the part of road surface or not. Different scenarios are appears for shadows or non shadow conditions. Accordingly, they propose a novel approach to vision-based road detection that is robust to shadows. The proposed algorithm works in still images and does not depend on either road shape or temporal restrictions. Quantitative and qualitative experiments on realworld road sequences with heavy traffic and shadows show that the method is robust to shadows and lighting variations. Moreover, the proposed method provides the highest performance when compared with hue-saturation-intensity (HSI)-based algorithms.

\section{CONCLUSION}

This paper shows the review of previously used method. Some of the methods provide good solutions in various weather conditions for detection of vehicles on the road lane. Most of the methods only produced the output only at day time. If overlapped vehicles or traffic is found on the road, most of the methods separate out single vehicle and detects the vehicles. Every method has their own identity and tries to produce good output. Finally Table 1 summarise how the used methods in previous research produces results in different conditions. 
Table 1. Different methods with the various weather conditions, vehicles detected in jamming or not and the disadvantage of that method. This symbol ' ', indicates not able to finding out disadvantage.

\begin{tabular}{|c|c|c|c|c|c|c|c|}
\hline \multirow[b]{2}{*}{ Method } & \multicolumn{4}{|c|}{ Weather Condition Mentioned } & \multirow{2}{*}{$\begin{array}{c}\text { Vehicles } \\
\text { detected in } \\
\text { Jamming } \\
\end{array}$} & \multirow{2}{*}{$\begin{array}{l}\text { Shadow } \\
\text { effect } \\
\text { remove }\end{array}$} & \multirow[b]{2}{*}{ Disadvantage } \\
\hline & Day & Night & Cloudy & Rainy & & & \\
\hline JRF Model[1] & Yes & Yes & Yes & Yes & Yes & Yes & $\begin{array}{l}\text { Do not classify vehicles \& } \\
\text { not recognize velocity. }\end{array}$ \\
\hline $\begin{array}{ll}\text { Colour } & \text { Transform } \\
\text { Model[2] } & \end{array}$ & Yes & Yes & Yes & Yes & No & No & $\begin{array}{l}\text { It did not address } \\
\text { resolution on traffic jams } \\
\text { and shadow reductions }\end{array}$ \\
\hline $\begin{array}{l}\text { multilevel framework to } \\
\text { detect and handle vehicle } \\
\text { occlusion[3] }\end{array}$ & Yes & Yes & Yes & Yes & Yes & Yes & -- \\
\hline $\begin{array}{l}\text { Detection } \\
\text { Classification of } \\
\text { Lanes Using } \\
\text { Motion Trajectories [5]. }\end{array}$ & Yes & No & No & No & $\begin{array}{l}\text { Partial or } \\
\text { complete } \\
\text { occlusion }\end{array}$ & Yes & $\begin{array}{l}\text { Vehicle detects if the } \\
\text { distance between vehicle } \\
\text { and the camera is less } \\
\text { otherwise it faces lots of } \\
\text { problems. }\end{array}$ \\
\hline $\begin{array}{ll}\text { Background } & \text { Estimation } \\
\text { Algorithm [6]. } & \\
\end{array}$ & Yes & Yes & No & No & Yes & Yes & - \\
\hline 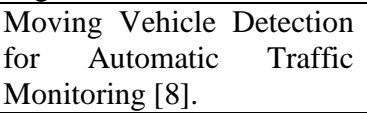 & Yes & Yes & Yes & Yes & Yes & Yes & $\begin{array}{l}\text { Separation of Overlapped } \\
\text { vehicles is not done. }\end{array}$ \\
\hline $\begin{array}{l}\text { Image Analysis and Rule- } \\
\text { Based Reasoning for a } \\
\text { Traffic } \\
\text { System[9]. Monitoring }\end{array}$ & Yes & Yes & No & No & Yes & No & $\begin{array}{l}\text { The reduction of shadow } \\
\text { effect is not the part of } \\
\text { defined method. }\end{array}$ \\
\hline $\begin{array}{l}\text { Road Detection Based on } \\
\text { Illuminant Invariance }\end{array}$ & Yes & No & No & No & No & Yes & $\begin{array}{l}\text { The goal of this method is } \\
\text { to detect road not vehicle } \\
\text { so it does not handle } \\
\text { overlapped vehicles. }\end{array}$ \\
\hline $\begin{array}{l}\text { Adaptive Vehicle detector } \\
\text { approach in complex } \\
\text { environments[10]. }\end{array}$ & Yes & Yes & Yes & Yes & Yes & Yes & $\begin{array}{l}\text { It does not detect vehicles } \\
\text { accurately in traffic jams } \\
\text { and all weather } \\
\text { conditions. }\end{array}$ \\
\hline
\end{tabular}

\section{REFERENCES}

[1] Y. Wang, "Joint random field model for all-weather moving vehicle detection," IEEE Trans.Image Process, vol. 19, no. 9, pp. 2491-2501,Sep. 2010.

[2] L.-W. Tsai, J.-W. Hsieh and K.-C. Fan, "Vehicle detection using normalized color and edge map," IEEE Trans. Image Process., vol. 16, no. 3,pp. 850-864, Mar. 2007.

[3] W. Zhang, Q. M. J.Wu, and X. Yang, "Multilevel framework to detect and handle vehicle occlusion," IEEE Trans. Intell. Transp. Syst., vol. 9, no. 1,pp. 161-174, Mar. 2008.

[4] N. K. Kanhere and S. T. Birchfield, "Real-time incremental segmentation and tracking of vehicles at low camera angles using stable features," IEEE Trans. Intell. Transp. Syst., vol. 9, no. 1, pp. 148-160, Mar. 2008.

[5] Melo, A. Naftel, A. Bernardino, and J. Santos-Victor, "Detection and classification of highway lanes using vehicle motion trajectories," IEEE Trans. Intell. Transp. Syst., vol. 7, no. 2, pp. 188-200, Jun. 2006.
[6] H.-Y. Cheng, B.-S. Jeng, P.-T. Tseng, and K.-C. Fan, "Lane detection with moving vehicles in the traffic scenes," IEEE Trans. Intell. Transp. Syst.,vol. 7, no. 4, pp. 571-582, Dec. 2006.

[7] M. Vargas, J. M. Milla, S. L. Toral, and F. Barrero, "An enhanced background estimation algorithm for vehicle detection in urban traffic scenes,"IEEE Trans. Veh. Technol., vol. 59, no. 8, pp. 3694-3709, Oct. 2010.

[8] J. Zhou, D. Gao, and D. Zhang, "Moving vehicle detection for automatic traffic monitoring," IEEE Trans. Veh. Technol., vol. 56, no. 1, pp. 51-59, Jan. 2007.

[9] R. Cucchiara, M. Piccardi, and P. Mello, "Image analysis and rule-based reasoning for a traffic monitoring system," IEEE Trans. Intell. Transp.Syst., vol. 1, no. 2, pp. 119-130, Jun. 2000.

[10] Bing-Fei Wu, Fellow, IEEE, and Jhy-Hong Juang,"Adaptive vehicle detector approach for complex envoirnmets," Trans. Intell. Transp.Syst., vol. 13, no. 2, pp. 817-827, Jun. 2012. 